\title{
Periodicity of solutions of nonhomogeneous linear difference equations
}

\author{
Klara Janglajew and Ewa Schmeidel
}

"Correspondence:

eschmeidel@math.uwb.edu.pl Institute of Mathematics, University of Białystok, Białystok, Poland

\begin{abstract}
Firstly, sufficient conditions for nonexistence of an $\omega$-periodic solution of the equation $x(n+1)+a_{0}(n) x(n)=b(n)$ are presented. Then, sufficient conditions under which every solution of the above equation is asymptotically $\omega$-periodic are given. Next, the results obtained for the first-order difference equation are generalized for the higher-order nonhomogeneous linear difference equation

$$
\sum_{i=0}^{k} a_{i}(n) x(n+i)=b(n)
$$

Finally, the periodic and asymptotically periodic solutions of this equation are investigated. Many examples illustrate the results given.
\end{abstract}

MSC: 39A11;39A10

Keywords: nonhomogeneous linear difference equation; asymptotically periodic solution

\section{Introduction}

We consider a class of $k$-order linear difference equations of the form

$$
\sum_{i=0}^{k} a_{i}(n) x(n+i)=b(n), \quad n \in \mathbf{N}=\{0,1,2, \ldots\},
$$

where $a_{0}(n) \neq 0, a_{k}(n) \neq 0$ for each $n \in \mathbf{N}$.

For the reader's convenience, we note that the background for difference equations theory can be found, e.g., in the well-known monograph by Agarwal [1] as well as in those by Elaydi [2], Kelley and Peterson [3] or Kocić and Ladas [4].

The investigation of linear difference equations attracted the attention of many mathematicians. Agarwal and Popenda, in [5], set together various basic statements on the periodicity of the solutions of first-order linear difference equations. In [6], the same authors studied periodic oscillation of solutions of nonhomogeneous higher-order difference equations. Popenda and Schmeidel (see [7]) considered the linear difference equation $c_{n}^{r} y_{n+r}+\cdots+c_{n}^{1} y_{n+1}+c_{n}^{0} y_{n}=d_{n}$ and presented sufficient conditions for the existence of an asymptotically constant solution of the above equation. In [8], the conditions which guarantee that the linear difference equation $x_{n+1}-a_{n} x_{n}=\sum_{i=0}^{r} a_{n}^{(i)} x_{n+i}$ possesses an asymptotically periodic solution were given by the same authors. In [9], Popenda and Schmeidel 
studied the linear difference equation, where one of the coefficients is periodic or constant and the others asymptotically approach zero, and obtained sufficient conditions for the existence of asymptotically periodic solutions. Smith (see [10]) investigated oscillatory and asymptotic behavior of solutions of linear third-order difference equations. In [11], asymptotic behavior of solutions of a linear second-order difference equation was studied by Trench.

For convenience, we adopt the notation for sequences $b=(b(n))$ and $a_{i}=\left(a_{i}(n)\right)$, where $i=0,1,2, \ldots, k$. Throughout this paper, we assume that $\sum_{n=k}^{l} a(n)=0$ and $\prod_{n=k}^{l} a(n)=1$ for $l<k$.

We begin with the following basic well-known definition.

Definition 1 The sequence $y: \mathbf{N} \rightarrow \mathbf{R}$ is called $\omega$-periodic if $y(n+\omega)=y(n)$ for all $n \in \mathbf{N}$. The sequence $y$ is called asymptotically $\omega$-periodic if there exist two sequences $u, v: \mathbf{N} \rightarrow$ $\mathbf{R}$ such that $u$ is $\omega$-periodic, $\lim _{n \rightarrow \infty} v(n)=0$, and $y(n)=u(n)+v(n)$ for all $n \in \mathbf{N}$.

It is clear that every constant function is 1-periodic.

If a sequence $a_{0}$ is $\omega_{1}$-periodic and $b$ is $\omega_{2}$-periodic in (1), then throughout this paper, $\omega$ is the least common multiple of $\omega_{1}$ and $\omega_{2}\left(\omega=\operatorname{lcm}\left(\omega_{1}, \omega_{1}\right)\right)$.

In the paper, we are looking for the periodic solutions of (1) with the period less than or equal to $\omega$. We are not interested in the solutions of (1) with the period greater than $\omega$, but such solutions can exist.

Example 1 The general solution of

$$
x(n+4)-x(n)=0
$$

is given by

$$
x(n)=c^{*}+c^{* *}(-1)^{n}+C^{*} \cos \left(n \frac{\pi}{2}\right)+C^{* *} \sin \left(n \frac{\pi}{2}\right) .
$$

Here, sequences $a_{0} \equiv 1$ and $b \equiv 0$ are 1-periodic, but there are 4-periodic solutions.

\section{First-order difference equations}

Periodicity of solutions of first-order linear nonhomogeneous difference equations was considered by Agarwal and Popenda in [5]. The authors contemplate the class of equations which have the same periodic solutions.

Let $k=1$ in equation (1) and $a_{1} \equiv 1$. Hence, equation (1) takes the following form:

$$
x(n+1)+a_{0}(n) x(n)=b(n) .
$$

If $a_{0} \equiv-1, b \equiv 0$, then the general solution of (2) is a constant function, then it is 1periodic.

If $a_{0} \equiv-1, b \neq \equiv$, then the general solution of (2) is

$$
x(n)=c+\sum_{j=0}^{n-1} b(j)
$$


where $c$ is an arbitrary constant. From (3) we see that a necessary and sufficient condition for the existence of $\omega$-periodic solutions of (2) is $b$ being an $\omega$-periodic sequence such that

$$
\sum_{j=0}^{\omega-1} b(j)=0
$$

The general solution of the associated homogeneous equation of (2) is

$$
x(n)=c(-1)^{n} \prod_{i=0}^{n-1} a_{0}(i) .
$$

If $a_{0}(n) \neq 0$ for any $n \in \mathbf{N}$, then the necessary and sufficient condition for the existence of a nontrivial $\omega$-periodic solution of the homogeneous equation is that $a_{0}$ is an $\omega$-periodic sequence and

$$
(-1)^{\omega} \prod_{i=0}^{\omega-1} a_{0}(i)=1 .
$$

If these conditions are satisfied, then all the solutions of the homogeneous equation are $\omega$-periodic. We also note that if $a_{0}(n)=0$, for some $n \in \mathbf{N}$, then $x \equiv 0$ for large enough $n$, and this solution is eventually a 1 -periodic solution.

From (2) we see that if $a_{0}$ is $\omega$-periodic, then the necessary condition for the existence of an $\omega$-periodic solution is $\omega$-periodicity of the sequence $b$.

Example 2 Consider the equation

$$
x(n+1)+\left(2+(-1)^{n}\right) x(n)=5+(-1)^{n} .
$$

Sequences $a_{0}(n)=2+(-1)^{n}$ and $b(n)=5+(-1)^{n}$ are 2-periodic. The solution $x(n)=$ $2+(-1)^{n+1}$ of the above equation is 2-periodic, too. Notice that there are not 2-periodic solutions of the associated homogeneous equation.

The following example shows us that in the case $a_{0}$ is $\omega$-periodic, $\omega$-periodicity of the sequence $b$ is not sufficient for the existence of an $\omega$-periodic solution of (2).

Example 3 Take in (2)

$$
a_{0}(2 n-1)=2, \quad a_{0}(2 n)=\frac{1}{2}, \quad b(2 n-1)=-2, \quad b(2 n)=1 .
$$

Sequences $a_{0}$ and $b$ are 2-periodic sequences. The general solution of the above equation

$$
\left\{\begin{array}{l}
x(2 n-1)=c-3+2 n \\
x(2 n)=-2 c+4-4 n,
\end{array}\right.
$$

is not a periodic sequence. 
Theorem 1 Let $a_{0}$ and $b$ be $\omega$-periodic in (2). The following statements then hold true:

(i) If

$$
(-1)^{\omega} \prod_{i=0}^{\omega-1} a_{0}(i) \neq 1
$$

then (2) has an w-periodic solution with the initial condition

$$
x(0)=\left(1-(-1)^{\omega} \prod_{i=0}^{\omega-1} a_{0}(i)\right)^{-1}\left[\sum_{j=0}^{\omega-1}\left((-1)^{\omega-j-1} \prod_{i=j+1}^{\omega-1} a_{0}(i)\right) b(j)\right] .
$$

(ii) If

$$
(-1)^{\omega} \prod_{i=0}^{\omega-1} a_{0}(i)=1, \quad \sum_{j=0}^{\omega-1}\left((-1)^{\omega-j-1} \prod_{i=j+1}^{\omega-1} a_{0}(i)\right) b(j)=0,
$$

then every solution of (2) is $\omega$-periodic.

(iii) If

$$
(-1)^{\omega} \prod_{i=0}^{\omega-1} a_{0}(i)=1, \quad \sum_{j=0}^{\omega-1}\left((-1)^{\omega-j-1} \prod_{i=j+1}^{\omega-1} a_{0}(i)\right) b(j) \neq 0,
$$

then there is no $\omega$-periodic solution of (2).

Proof The solution of equation (2) is given by

$$
x(n)=\left((-1)^{n} \prod_{i=0}^{n-1} a_{0}(i)\right) x(0)+\sum_{j=0}^{n-1}\left((-1)^{n-j-1} \prod_{i=j+1}^{n-1} a_{0}(i)\right) b(j) .
$$

From the above, the result follows immediately.

Assume that condition (4) holds. It follows from (i) that equation (2) has a unique $\omega$ periodic solution if and only if the homogeneous equation

$$
x(n+1)+a_{0}(n) x(n)=0
$$

has not any nontrivial $\omega$-periodic solution.

In [5] Agarwal and Popenda proved that if $a_{0}$ is not periodic, then equation (2) can have at most one periodic solution.

Example 4 The equation

$$
x(n+1)+\frac{1}{2^{n}} x(n)=(-1)^{n+1}\left(1-\frac{1}{2^{n}}\right)
$$

has a unique periodic solution $x(n)=(-1)^{n}$. Here, the general solution

$$
x(n)=c(-1)^{n-1} 2^{-\frac{n(n-1)}{2}}
$$

of the associated homogeneous equation has not any nontrivial periodic solution. 
The following example shows us that there exists a class of equations (2) which have the same $\omega$-periodic solutions (each of them differs on the subsequence $\left(a_{0}(3 n-1)\right)$ ).

Example 5 Let $a_{0}=(3,-1, a, 3,-1, a, \ldots)=(3,-1, a)_{3}, b=(2,4,2)_{3}$. It is easy to check that the sequence $x=(2,-4,0)_{3}$ is a 3 -periodic solution of (2) independently of the values taken for $a$.

This leads to the problem of defining the class of equations which have the same periodic solutions.

Let $a_{0}^{\prime \prime}$ be an $\omega$-periodic sequence which fulfills condition (4) and $p \in\{0,1,2, \ldots, \omega-1\}$. We define the set $S_{p}$ as follows:

$$
S_{p}=\left\{a_{0}^{*}=\left\{a_{0}^{*}(n)\right\}: a_{0}^{*}(i)=a_{0}(i) \text { for } i \neq p, a_{0}^{*}(i) \neq a_{0}(i) \text { for } i=p\right\} .
$$

Theorem 2 Assume that in equation (2) sequences $a_{0}$ and $b$ are $\omega$-periodic and condition (4) holds.

If

$$
\begin{aligned}
& \left(1-(-1)^{\omega} \prod_{i=0}^{\omega-1} a_{0}(i)\right)\left(1-(-1)^{\omega} \prod_{i=0}^{\omega-1} a_{0}^{*}(i)\right)^{-1} \\
& =\left[\sum_{j=0}^{\omega-1}\left((-1)^{\omega-j-1} \prod_{i=j+1}^{\omega-1} a_{0}(i)\right) b(j)\right]\left[\sum_{j=0}^{\omega-1}\left((-1)^{\omega-j-1} \prod_{i=j+1}^{\omega-1} a_{0}^{*}(i)\right) b(j)\right]^{-1}
\end{aligned}
$$

and

$$
\begin{aligned}
& (-1)^{p} \prod_{i=0}^{p-1} a_{0}(i)\left[\sum_{j=0}^{\omega-1}\left((-1)^{\omega-j-1} \prod_{i=j+1}^{\omega-1} a_{0}(i)\right) b(j)\right]\left(1-(-1)^{\omega} \prod_{i=0}^{\omega-1} a_{0}(i)\right)^{-1} \\
& +\sum_{j=0}^{p-1}\left((-1)^{\omega-j-1} \prod_{i=j+1}^{p-1} a_{0}(i)\right) b(j)=0
\end{aligned}
$$

then every equation of the form

$$
x(n+1)+a_{0}^{*}(n) x(n)=b(n), \quad a_{0}^{*} \in S_{p},
$$

has the same $\omega$-periodic solution $x$ as equation (2) independently on $a_{0}^{*}(p)$ term.

Proof Let $x$ and $x^{*}$ be the solutions of equations (2) and (9) respectively. The assumptions of Theorem 1 hold for equations (2) and (9), then by (5) and (7), we get that $x(0)=x^{*}(0)$. Because $a_{0}(i)=a_{0}^{*}(i)$ for $i=0,1,2, \ldots, p-1$, we get $x(i)=x^{*}(i)$ for $i=0,1,2, \ldots, p-1$. From (6), (8), and $x(0)=x^{*}(0)$, we have $x(p)=x^{*}(p)=0$. So, $x(i)=x^{*}(i)$ for $i=p, p+1, \ldots, \omega-1$. By $\omega$-periodicity of $x$ and $x^{*}, x=x^{*}$.

Now, we turn our attention to asymptotical periodicity of the solutions of (2). 
Assume that $a_{0}$ is $\omega$-periodic, $b(n)=c(n)+d(n)$, where $c$ is $\omega$-periodic and $\lim _{n \rightarrow \infty} d(n)=$ 0 . Let $y$ be a solution of the equation

$$
y(n+1)+a_{0}(n) y(n)=c(n)
$$

and $z$ be a solution of the equation

$$
z(n+1)+a_{0}(n) z(n)=d(n) .
$$

Hence, $x=y+z$ is a solution of

$$
x(n+1)+a_{0}(n) x(n)=c(n)+d(n) .
$$

Set $a_{0}(n)=-\alpha_{0}(n)$. Multiplying both sides of equation (10) by $\prod_{i=0}^{n-\omega\left[\frac{n}{\omega}\right]} \frac{1}{\alpha_{0}(i)}$, we obtain

$$
\prod_{i=0}^{n-\omega\left[\frac{n}{\omega}\right]} \frac{1}{\alpha_{0}(i)} z(n+1)-\prod_{i=0}^{n-1-\omega\left[\frac{n-1}{\omega}\right]} \frac{1}{\alpha_{0}(i)} z(n)=\prod_{i=0}^{n-\omega\left[\frac{n}{\omega}\right]} \frac{1}{\alpha_{0}(i)} d(n) .
$$

Summing the above equality from $j=0$ to $n-1$, we obtain

$$
\begin{aligned}
& \sum_{j=0}^{n-1}\left(\prod_{i=0}^{j-\omega\left[\frac{j}{\omega}\right]} \frac{1}{\alpha_{0}(i)} z(j+1)-\prod_{i=0}^{j-1-\omega\left[\frac{j-1}{\omega}\right]} \frac{1}{\alpha_{0}(i)} z(j)\right) \\
& \quad=\sum_{j=0}^{n-1}\left(\prod_{i=0}^{j-\omega\left[\frac{j}{\omega}\right]} \frac{1}{\alpha_{0}(i)} d(j)\right),
\end{aligned}
$$

and

$$
\sum_{j=0}^{n-1}\left[\Delta\left(\prod_{i=0}^{j-1-\omega\left[\frac{j-1}{\omega}\right]} \frac{1}{\alpha_{0}(i)} z(j)\right)\right]=\sum_{j=0}^{n-1}\left(\prod_{i=0}^{j-\omega\left[\frac{j}{\omega}\right]} \frac{1}{\alpha_{0}(i)} d(j)\right)
$$

Hence,

$$
\left(\prod_{i=0}^{n-1-\omega\left[\frac{n-1}{\omega}\right]} \frac{1}{\alpha_{0}(i)}\right) z(n)=z(0) \prod_{i=0}^{-1-\omega\left[\frac{-1}{\omega}\right]} \frac{1}{\alpha_{0}(i)}+\sum_{j=0}^{n-1}\left(\prod_{i=0}^{j-\omega\left[\frac{j}{\omega}\right]} \frac{1}{\alpha_{0}(i)}\right) d(j)
$$

Assuming

$$
\sum_{j=0}^{\infty}\left(\prod_{i=0}^{j-\omega\left[\frac{j}{\omega}\right]} \frac{1}{\alpha_{0}(i)}\right) d(j)+z(0) \prod_{i=0}^{-1-\omega\left[\frac{-1}{\omega}\right]} \frac{1}{\alpha_{0}(i)}=S
$$

and letting $n \rightarrow \infty$, the right side of the above equality tends to some constant $c=z(0)+S$, then the left one does too. Utilizing little- $o$ notation, we obtain

$$
\left(\prod_{i=0}^{n-1-\omega\left[\frac{n-1}{\omega}\right]} \frac{1}{\alpha_{0}(i)}\right) z(n)=c+o(1)
$$


Hence,

$$
x(n)=y(n)+c\left(\prod_{i=0}^{n-1-\omega\left[\frac{n-1}{\omega}\right]}\left(-a_{0}(i)\right)\right)+o(1)\left(\prod_{i=0}^{n-1-\omega\left[\frac{n-1}{\omega}\right]}\left(-a_{0}(i)\right)\right) .
$$

From above, we get sufficient conditions for asymptotical periodicity of the solutions of (2) which are presented in the following theorem.

Theorem 3 Let the sequence $a_{0}$ be $\omega$-periodic and $b(n)=c(n)+d(n)$, where $c$ is $\omega$-periodic and the series

$$
\sum_{j=0}^{\infty}\left(\prod_{i=0}^{j-\omega\left[\frac{j}{\omega}\right]} \frac{-1}{a_{0}(i)}\right) d(j)
$$

converges, then there exists an asymptotically $\omega$-periodic solution of equation (2). Moreover, if conditions

$$
(-1)^{\omega} \prod_{i=0}^{\omega-1} a_{0}(i)=1, \quad \sum_{j=0}^{\omega-1}\left((-1)^{\omega-j} \prod_{i=j+1}^{\omega-1} a_{0}(i)\right) c(j)=0
$$

hold, then every solution of equation (2) is asymptotically $\omega$-periodic.

The following three examples illustrate the result presented in Theorem 3:

- in the first one sequence $a_{0}$ is constant;

- in the second sequence $a_{0}$ is 3-periodic;

- in the third sequence $a_{0}$ is not periodic.

Example 6 Consider the equation

$$
x(n+1)-x(n)=2(-1)^{n} .
$$

The assumptions of Theorem 2 hold $\left(c(n)=2(-1)^{n}, d(n)=0\right)$. The general solution of the equation $x(n)=c+(-1)^{n+1}$ is an asymptotically 2 -periodic sequence.

Example 7 Assume that

$$
a_{0}(3 n-2)=2, \quad a_{0}(3 n-1)=\frac{1}{2}, \quad a_{0}(3 n)=-1,
$$

in $(2)$ and $b(n)=c(n)+d(n)$, where

$$
\begin{aligned}
& c(3 n-2)=2, \quad c(3 n-1)=0, \quad c(3 n)=1, \\
& d(3 n-2)=8^{-n}, \quad d(3 n-1)=\frac{5}{4} 8^{-n}, \quad d(3 n)=-\frac{31}{32} 8^{-n} .
\end{aligned}
$$

Furthermore,

$$
\sum_{j=1}^{n}\left|\prod_{i=1}^{j} \frac{1}{a_{0}(i)} d(j)\right| \leq \frac{5}{2} \sum_{j=1}^{n} 8^{-j}
$$


Hence,

$$
\sum_{j=1}^{\infty}\left|\prod_{i=1}^{j} \frac{1}{a_{0}(i)} d(j)\right|<\infty
$$

All the assumptions of Theorem 2 are satisfied. Therefore, all the solutions of equation (2) are asymptotically 3-periodic. This can be easily seen from the general solution of the considered equation, which is given below.

$$
\left\{\begin{array}{l}
x(3 n-2)=c+2+\frac{1}{4} \cdot 8^{-n} \\
x(3 n-1)=-2 c-2+\frac{1}{2} \cdot 8^{-n} \\
x(3 n)=c+1+8^{-n}
\end{array}\right.
$$

Example 8 Let us put $a_{0}(n)=-\frac{1}{n+1}, b(3 n-2)=\frac{6 n-3}{3 n-1}, b(3 n-1)=\frac{9 n-2}{3 n}, b(3 n)=\frac{3 n-2}{3 n+1}$ in $(2)$. Hence, the general solution of the associated homogeneous equation

$$
x(n)=\frac{c}{(n-1) !}
$$

tends to zero. The 3 -periodic solution of $(2)$ is $x=\left((1,2,3)_{3}\right)$. Therefore, every solution of (2) is asymptotically 3 -periodic.

\section{Some results for higher-order equations}

In this part, we study equation (1). In the following theorem, sufficient conditions under which equation (1) has no asymptotically periodic solution are given.

Theorem 4 Assume that there exists $i_{0} \in\{0,1,2, \ldots, k\}$ such that $\sup _{n \in \mathbf{N}}\left|a_{i_{0}}(n)\right|=\infty$ and $\sup _{n \in \mathbf{N}}\left|a_{j}(n)\right|<\infty$ for $j \neq i_{0}, j \in\{0,1,2, \ldots, k\}$. Let the sequence $b$ be bounded, too. Then equation (1) has not any asymptotically periodic solution $x: \mathbf{N} \rightarrow \mathbf{R} \backslash\{0\}$.

Proof Suppose to the contrary that (1) has such an asymptotically periodic solution $x$. It implies that the sequence $x$ is bounded. Choose $i_{0} \in\{0,1,2, \ldots, k\}$ such that the sequence $\left(a_{i_{0}}(n)\right)$ is unbounded. Therefore,

$$
a_{i_{0}}(n) x\left(n+i_{0}\right)
$$

is also unbounded. Hence,

$$
\sum_{i=0}^{k} a_{i}(n) x(n+i)
$$

is unbounded, while $b$ is bounded. This contradiction completes the proof.

The sufficient conditions for the existence of an asymptotically $\omega$-periodic solution of equation (1) are given in the following theorem. 
Theorem 5 Assume that $a_{0}: \mathbf{N} \rightarrow \mathbf{R} \backslash\{0\}$, the condition

$$
\lim _{n \rightarrow \infty} \frac{a_{i}(n)}{a_{0}(n)}=0
$$

holds for each $i \in\{1,2, \ldots, k\}$ and the sequence $\left(\frac{b(n)}{a_{0}(n)}\right)$ is asymptotically $\omega$-periodic. Then there exists an asymptotically $\omega$-periodic solution of equation (1).

Proof From the periodicity of the sequence $\left(\frac{b(n)}{a_{0}(n)}\right)$, there exists a positive constant $C$ such that

$$
\left|\frac{b(n)}{a_{0}(n)}\right|<C \text {. }
$$

From condition (11), for any $\varepsilon>0$, there exists a positive integer $N$ such that

$$
\sum_{i=1}^{k}\left|\frac{a_{i}(n)}{a_{0}(n)}\right|<\varepsilon \quad \text { for } n \geq N .
$$

Set $c=C+\varepsilon$. We define the sequence $\alpha$ as follows:

$$
\alpha(n)=\sum_{i=1}^{k}\left|\frac{a_{i}(n)}{a_{0}(n)}\right| .
$$

Let $B_{N}$ be the Banach space of all real bounded sequences $x$ defined for $n \geq N$, with usually 'sup' norm. Set

$$
S=\left\{x(n) \in B_{N}: \frac{b(n)}{a_{0}(n)}-c \alpha(n) \leq x(n) \leq \frac{b(n)}{a_{0}(n)}+c \alpha(n) \text { for } n \geq N\right\} .
$$

It is not difficult to prove that $S$ is a nonempty, closed, convex, and compact subset of $B_{N}$. For example, to show that the set $S$ is convex, let us take sequences $x, y \in S$ and a real constant $\beta \in[0,1]$. Thus, multiplying (12) by $\beta$, we obtain

$$
\beta \frac{b(n)}{a_{0}(n)}-c \beta \alpha(n) \leq \beta x(n) \leq \beta \frac{b(n)}{a_{0}(n)}+c \beta \alpha(n) .
$$

Analogously, for the sequence $y$, we have

$$
(1-\beta) \frac{b(n)}{a_{0}(n)}-c(1-\beta) \alpha(n) \leq(1-\beta) y(n) \leq(1-\beta) \frac{b(n)}{a_{0}(n)}+c(1-\beta) \alpha(n) .
$$

Summing the above inequalities, we get

$$
\frac{b(n)}{a_{0}(n)}-c \alpha(n) \leq \beta x(n)+(1-\beta) y(n) \leq \frac{b(n)}{a_{0}(n)}+c \alpha(n) \quad \text { for } n \geq N .
$$

It means that the set $S$ is convex. 
Let us define a mapping $T: S \rightarrow B_{N}$ as follows:

$$
(T x)(n)=\frac{b(n)}{a_{0}(n)}-\sum_{i=1}^{k} \frac{a_{i}(n)}{a_{0}(n)} x(n+i) \quad \text { for } n \geq N .
$$

We show that $T(S) \subset S$. Indeed, if $x \in S$, then $|x(i)|<c$ for large $n>N$, and

$$
\left|(T x)(n)-\frac{b(n)}{a_{0}(n)}\right| \leq c \sum_{i=1}^{k}\left|\frac{a_{i}(n)}{a_{0}(n)}\right|=c \alpha(n) .
$$

We see that $T$ is continuous. Hence, by the Schauder fixed point theorem, there exists $x \in S$ such that $x(n)=(T x)(n)$ for $n \geq N$, so

$$
x(n)=\frac{b(n)}{a_{0}(n)}-\sum_{i=1}^{k} \frac{a_{i}(n)}{a_{0}(n)} x(n+i) \quad \text { for } n \geq N,
$$

hence it is a solution of equation (1). Because $x \in S$, then $x$ is an asymptotically periodic sequence. This completes the proof.

Example 9 Consider the equation

$$
\begin{aligned}
& \frac{1}{(n+2)(n+3)} x(n+3)+\frac{1}{n+2} x(n+2)+\frac{1}{n+1} x(n+1)+x(n) \\
& =(-1)^{n}+(-1)^{n} \frac{2 n^{2}+7 n+4}{n(n+2)^{2}}+(-1)^{n+1} \frac{n^{4}+11 n^{3}+43 n^{2}+69 n+40}{(n+1)^{2}(n+2)(n+3)^{2}} .
\end{aligned}
$$

By Theorem 5, we get that there exists an asymptotically periodic solution of the above equation. In fact, the asymptotically 2 -periodic sequence

$$
x(n)=(-1)^{n}+(-1)^{n} \frac{1}{n}
$$

is such a solution.

\section{Competing interests}

The authors declare that they have no competing interests.

\section{Authors' contributions}

The authors have achieved equal contributions to each part of this paper. All authors read and approved the final manuscript.

Received: 17 July 2012 Accepted: 3 November 2012 Published: 14 November 2012

\section{References}

1. Agarwal, RP: Difference Equations and Inequalities. Theory, Methods and Applications. Dekker, New York (2000)

2. Elaydi, SN: An Introduction to Difference Equations. Springer, New York (2005)

3. Kelley, WG, Peterson, AC: Difference Equations: An Introduction with Applications. Academic Press, San Diego (2001)

4. Kocic, VL, Ladas, G: Global Behavior of Nonlinear Difference Equations of Higher Order with Applications. Kluwer Academic, Dordrecht (1993)

5. Agarwal, RP, Popenda, J: Periodic solutions of first order linear difference equations. Math. Comput. Model. 22(1), 11-19 (1995)

6. Agarwal, RP, Popenda, J: On the oscillation of recurrence equations. Nonlinear Anal., Theory Methods Appl. 36(2), 231-268 (1999) 
7. Popenda, J, Schmeidel, E: On the asymptotic behaviour of nonhomogeneous linear difference equations. Indian J. Pure Appl. Math. 28(3), 319-327 (1997)

8. Popenda, J, Schmeidel, E: On the asymptotically periodic solution of some linear difference equations. Arch. Math. 35(1), 13-19 (1999)

9. Popenda, J, Schmeidel, E: Asymptotically periodic solution of some linear difference equations. Facta Univ. Ser. Math. Inform. 14, 31-40 (1999)

10. Smith, B: Linear third-order difference equations: oscillatory and asymptotic behavior. Rocky Mt. J. Math. 22(4), 1559-1564 (1992)

11. Trench, WF: Asymptotic behavior of solutions of a linear second-order difference equation. J. Comput. Appl. Math. 41(1-2), 95-103 (1992)

doi:10.1186/1687-1847-2012-195

Cite this article as: Janglajew and Schmeidel: Periodicity of solutions of nonhomogeneous linear difference equations. Advances in Difference Equations 2012 2012:195.

Submit your manuscript to a SpringerOpen ${ }^{\circ}$ journal and benefit from:

- Convenient online submission

- Rigorous peer review

- Immediate publication on acceptance

- Open access: articles freely available online

- High visibility within the field

- Retaining the copyright to your article

Submit your next manuscript at $\boldsymbol{~ s p r i n g e r o p e n . c o m ~}$ 